\title{
Erratum to: A new grading system of lumbar central canal stenosis on MRI: an easy and reliable method
}

\author{
Guen Young Lee $\cdot$ Joon Woo Lee $\cdot$ Hee Seok Choi • \\ Kyoung-Jin Oh $\cdot$ Heung Sik Kang
}

Published online: 12 April 2011

(C) ISS 2011

Erratum to: Skeletal Radiol (2010) 46 343-353

DOI 10.1007/s00256-011-1102-x

Author names were unfortunately published with errors.

Please find here correct list of authors.

The online version of the original article can be found at http://dx.doi. org/10.1007/s00256-011-1102-x.

G. Y. Lee $\cdot$ J. W. Lee $(\bowtie) \cdot$ H. S. Choi $\cdot$ K.-J. Oh $\cdot$ H. S. Kang

Department of Radiology,

Seoul National University Bundang Hospital,

300 Gumi-dong, Bundang-gu,

Seongnam-si, Gyeongi-do 464-707, South Korea

e-mail: joonwoo2@gmail.com

G. Y. Lee

e-mail: netty0523@hanmail.net

H. S. Choi

e-mail: drchs-1@hanmail.net

K.-J. Oh

e-mail: okradiology@gmail.com

H. S. Kang

e-mail: kanghs@radcom.snu.ac.kr 